\title{
Cloud Computing / Computación en nube
}

\author{
Isabel Maya Proaño*
}

\begin{abstract}
Resumen
El Cloud computing o computación en Nube, en la actualidad, constituye una excelente alternativa tecnológica para numerosas empresas; su radio de acción potencialmente alcanza a todo usuario informático del planeta. Este sistema está basado en la externalización de los recursos informáticos y puede ofrecer diversas aplicaciones y provisión de servicios a través de la Internet, eliminando dispositivos físicos.
\end{abstract}

\section{Palabras clave}

Computación nube - innovación tecnológica - gestión informática - topología de las comunicaciones - externalización informática

\begin{abstract}
Cloud computing today, technology is an excellent alternative for many companies, its range of potentially reaches every computer user on the planet. This system is based on the outsourcing of computing resources and can offer various applications and provision of services through the Internet, eliminating physical devices.
\end{abstract}

\section{Key words}

Cloud Computing - technological innovation - information management - communication topology - outsourcing computer

Forma sugerida de citar: Proaño Maya, Isabel. 2011. Cloud Computing/Computación en nube. Retos 1. Enero/Junio. Pp. 35-40.

* Docente de la Universidad Internacional SEK. Asesora del Ministerio de Relaciones Laborales del Ecuador. isa_mayal3@hotmail.com.

Retos I(I): 20II.

(c) 20II, Universidad Politécnica Salesiana del Ecuador 
La capacidad de procesamiento y almacenamiento, la movilidad y aplicabilidad de un equipo electrónico (VLSI)[1] en el mundo informático-digital marcan la tendencia y el horizonte del avance tecnológico. Se ratifica el enunciado, revisando el 'mejoramiento' que ha sufrido un equipo de sonido convencional, pasando por un walkman electrónico hasta llegar a un complejo, minúsculo y multifuncional 'iPod'. Enmarcado en las líneas de tendencia tecnológica mencionadas, encontramos a íconos empresariales como Microsoft, Cisco, McAfee, Oracle, Dell, Alcatel, entre otros, patrocinando el Tercer Foro gratuito sobre Cloud Computing, que tendrá lugar en junio de este año en Londres[2].

El internet es graficado como una nube en todo esquema topológico de comunicaciones y redes informáticas, se colige entonces que Cloud Computing está relacionado con el internet. Sin embargo, lo novedoso de este concepto radica en la externalización (virtualización en términos informáticos) de los recursos informáticos (hardware, software e infraestructura), pues estos radicarían en la nube (internet). Esta innovación tecnológica se perfila muy ambiciosa al brindar un esquema alternativo en la gestión de los recursos tradicionales de IT; cuya arquitectura pretende convertir el Internet en un 'servicio' como la luz, el teléfono o el agua potable. Se presenta conveniente para el individuo común que puede rentar espacio en internet para 'colgar'[3] su información y tenerla disponible en cualquier lugar que tenga acceso a este servicio. Como también para la pequeña y mediana empresa que puede alquilar a un tercero los servicios de procesamiento, administración y residencia de sus datos. Su estrategia es rentar espacio, equipos, plataformas y recursos a los clientes que así lo requieran, desvinculando al usuario del equipo (iPhone, blackberry) o computador que utilice. Definitivamente más conveniente que incurrir en gastos e inversiones relativamente altas en toda la infraestructura informática, dispositivos, plataformas, equipos, licencias y demás. Sin contar con el ahorro de capital humano, en mantenimiento de equipos, administración de la información y la red.

Los entendidos hablan de 'Cloud Computing' como un cambio de paradigma. Fernando de la Prida, responsable de la filial española de EMC, afirmó que: “...es un cambio de paradigma en la manera de entender la infraestructura. Pasamos de un concepto tradicional a entenderla como un servicio"; Ajel Gopal de CA Technology manifiesta: "No se trata de un nuevo avance. Cloud Computing representa un cambio de paradigma en el sector de las TI y en la forma en que la tecnología transformará los negocios". Larry Ellison CEO co-fundador de Oracle señala que “... en términos 


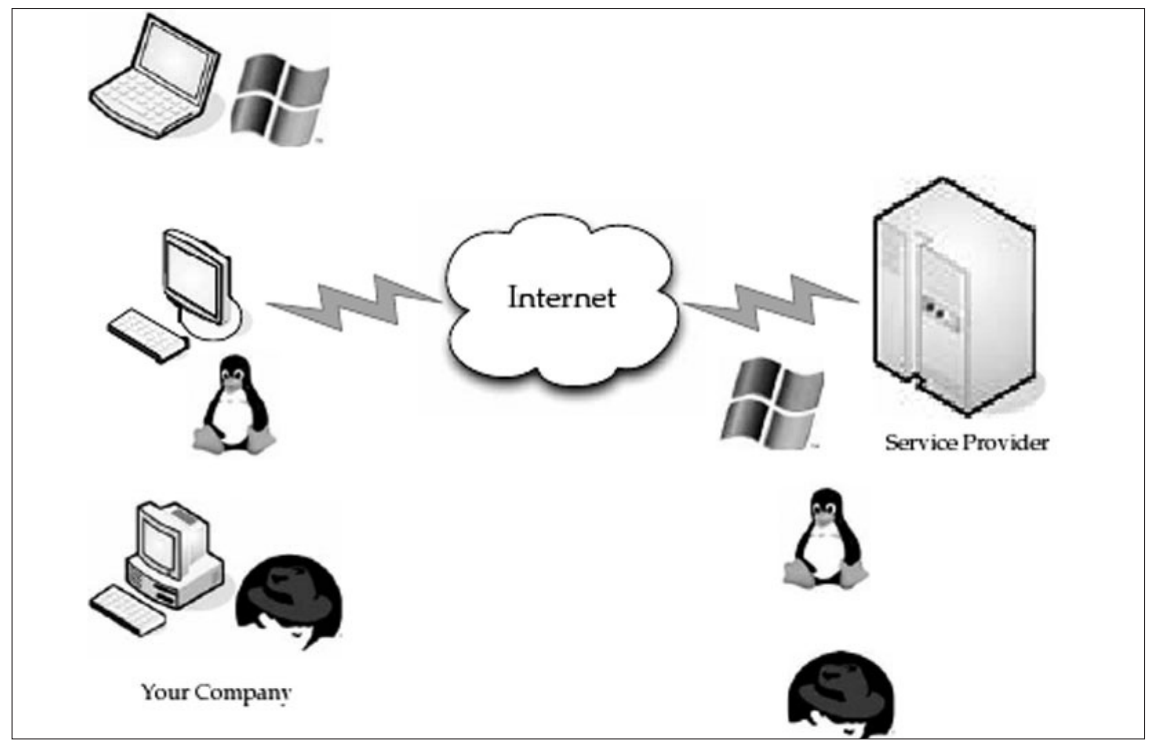

Gráfico 1. Esquema del Cloud Computing

de modelo de negocios es solamente el arrendamiento de recursos, que no involucra ninguna innovación tecnológica real, hemos estado realizando Cloud Computing por la pasada década".

Cualquiera que fuera el enfoque, queda claro que Cloud Computing marca un cambio de paradigma en la informática que radica en hacer de la internet un 'servicio', no solo una herramienta informativa. Es así, que los servicios que se ofertan en la red han sido estratificados bajo las abreviaturas SaaS (Software-as-a-Service), PaaS (Plataform-as-a-Service), HaaS (Hardware-as-a-Service).

Las empresas más reconocidas del mundo como IBM, Google, Amazon, Microsoft entre otras, es-

Retos I ( ): 20I I

(C) 20I I, Universidad Politécnica Salesiana del Ecuador tán apostando su inversión de millones de dólares en aras de ofrecer los mejores servicios Cloud. Prueba de esto es el producto google docs o hotmail Apps. Tal ha sido la inversión extranjera, que la firma de investigación Gartner Inc. estimó que los ingresos 'nube de servicios' de este año ascienden a 56 mil millones dólares, con un crecimiento real del $21 \%$ a partir de 2008 , a pesar de la crisis crediticia. Las previsiones del mercado cifran en 150 mil millones de dólares los ingresos en 2013.

\section{¿Qué significaría Cloud Computing para la pequeña y mediana industria?}

En primer lugar, un rubro de gastos basado en la entrega de servicios de IT puede tener una ventaja enorme de costo para la PYME. 


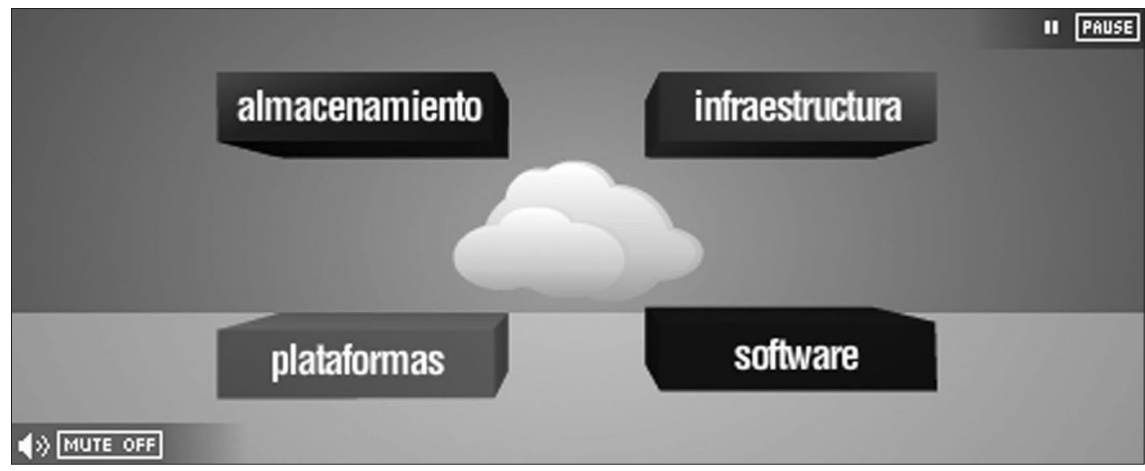

Gráfico 2. Oferta de servicios Cloud de IBM

Al evitar el gasto inicial de capital en sistemas complejos de infraestructura tecnológica, las empresas pueden ahorrar dinero y redireccionar estos recursos en potenciar otras áreas. La infraestructura informática se convierte ahora únicamente en un gasto de operación, ya que no hay hardware para comprar, administrar o hacer mantenimiento. El modelo Cloud también puede ayudar a las empresas a evitar el retorno de la inversión de riesgo y la incertidumbre, la posibilidad de cambio de proveedor si el servicio no funciona correctamente; evitar la pesadilla del fracaso costoso de activo fijo de seis meses en línea. Cloud Computing ofrece la eficiencia y la utilización de los recursos sobre la base de un pay-as-you-go (paga conforme utilices), modelo para una mejor escalabilidad. Las empresas pueden asignar fondos a los servicios —más o menos- en función de la cantidad que realmente necesitan, de crecimiento o reducción en el tiempo. Evitar gastos de capital inicial innecesarios también permite a las empresas levantarse del suelo y correr mucho más rápido que antes. Esto puede ser una ventaja para las empresas más pequeñas. Sin la barrera 'costo inicial' puede reducir notablemente el tiempo de comercialización.

La ventaja de la independencia de ubicación es otro de los beneficios clave de los servicios Cloud. Los avances de tercera generación (3G) y la tecnología inalámbrica de banda ancha han hecho el acceso a la Internet más fácil. Los usuarios pueden conectarse al instante a sus aplicaciones de software, sus datos almacenados y la información en la red. El resultado es la ausencia de infraestructura en la oficina. Para las empresas con múltiples usuarios en el campo, las aplicaciones no necesitan ser instaladas directamente en la máquina del usuario, lo que la hace funcionar con más eficiencia y seguridad. 
Algunos argumentan que el Cloud Computing nunca va a despegar porque los usuarios dependen de una conexión a Internet. La realidad es que, aunque la conexión a Internet no está disponible por doquier, es solo una cuestión de tiempo para que la tecnología evolucione y esto no sea un problema nunca más. El crecimiento de las ventas Dongle es un claro indicador de la demanda. Mientras tanto los proveedores de Cloud están buscando una forma de evitar eso. Google ya ha anticipado el retraso en la conectividad abierta y la preferencia para el cálculo de locales, haciendo algunas de sus aplicaciones descargables, característica que cubre todas las bases.

De lo expuesto, se puede determinar ciertos aspectos sensibles como la dependencia en el servicio en los medios de transmisión y la disponibilidad de los mismos, junto con un ideal tiempo de respuesta. La inseguridad en cuanto a integridad y privacidad de la información en términos de acceso fraudulento y en caso de contingencia física de los equipos. La centralización del proceso de información de una buena parte del aparato económico estaría sujeta a mayor control y mayor ataque delictivo (hackers, spyware, trojans). Aspectos que si no son disuasivos, deberían estar clara y debidamente absueltos y sentados en los respectivos contratos de servicio.

Retos I(I): 201 I

(c) 20II, Universidad Politécnica Salesiana del Ecuador
La fortaleza de Cloud Computing o Computación en la nube radica en la capacidad de procesamiento y de almacenamiento de datos, la movilidad y aplicabilidad de la misma, pues, si se contrata todo el servicio; esto es SaaS, PaaS y HaaS únicamente se necesitará acceder a cualquier 'cybercafe' del lugar donde se encuentre, ingresar a su información individual, realizar lo que necesita y luego pagar únicamente el tiempo de utilización del computador, algo muy ínfimo. Es un valor añadido real a las empresas pequeñas y medianas en todo el mundo, pues como observa Fernando de la Prida, responsable de la filial española de EMC, "De los presupuestos TI la gran compañía dedica el 75\% a mantener sus sistemas, equipos e infraestructuras y solo el 25\% va destinado a diseñar nuevos procesos y nuevas aplicaciones para el negocio. Ese cambio de chip es ser capaces de implantar soluciones de Cloud para poder reducir esas necesidades de gasto en mantenimiento, y utilizar ese presupuesto para innovar. Con la infraestructura en la nube se reducen costes, se generan puestos de trabajo y se genera un impacto en los ingresos de las compañías".

\section{Notas}

[1] Aparato que contiene circuitos integrados de alta densidad, como computadores, celulares, sistemas de control, etcétera.

[2] ‘www.cloudwf.com〉

[3] Término utilizado para referirse a guardar o mantener información en la Internet. 


\section{Bibliografía:}

Textos

Cloud Computing, A Practical Approach, Velte, Toby Velte, Anthony Elsepeter, Robert, publisher Mc Graw-Hill Professional Publishing, 09, 2009.

\section{REFERENCIAS DE INTERNET}

〈http://www.ibm.com/expressadvantage/cl/ olds/09/05/tn_showmail.phtml>, servicios Cloud Computing de IBM, Chile

< h t tp://www.computing.es/Entrevis tas/201104110006/INFRAESTRUCTU. RAS-En-EMC-no-buscamos-rentabilidaden-cloud-computing-sino-flexibilidadpara-el-cliente.aspx', Ajel Gopal de CA Technology, entrevista realizada por la revista Computing de España el 11 de abril de 2011.

< h t t p ://www.you tube.com/wat ch? $\mathrm{v}=$ OFacYAI6DY0, Larry Ellison, CEO y cofundador de Oracle, "What the hell is Cloud Computing".

〈http://referenzia.com/tag/foro-denae/Sönke Lund, publicado el 3 de noviembre de 2010, "Con el Cloud Computing estamos ante un cambio de paradigma".

〈http://www.techweek.es/infraestructuras-tic/ informes/1007680003701/cloud-computingcambiara-paradigma.1.html. Cloud Computing cambiará el paradigma de las TI en 2011, predice CA Tecnologies, 17 diciembre 2010.

〈http://www.cloudwf.com〉 Free 3rd Cloud Computing World Forum.

Envío 6 de febrero de 2011 - aprobación 25 de abril de 2011 